\title{
Efecto de la adición de biosólido (seco) a una pasta cerámica sobre la resistencia mecánica de ladrillos
}

\author{
William Mozo* \\ A Gómez ${ }^{* *}$ \\ Gloria Camargo***
}

Recibido: 22/11/2014 • Aceptado: 23/10/2015

\begin{abstract}
Resumen
Se adicionó biosólido como componente de una pasta cerámica para la fabricación de ladrillos. Se caracterizaron las materias primas (arcilla y biosólido) en cuanto a composición química, fases mineralógicas, comportamiento térmico y características físicas. Se fabricaron ladrillos con hasta un $15 \%$ de inclusión de biosólido en estado seco, de dimensiones estándar según Norma Técnica Colombiana (NTC) 296 y cocidos a temperaturas de $950{ }^{\circ} \mathrm{C}, 1000{ }^{\circ} \mathrm{C}$ y $1050{ }^{\circ} \mathrm{C}$. Se realizaron pruebas de resistencia a la compresión bajo los lineamientos de la NTC 4017, con el fin de evaluar el efecto que sobre esta propiedad tiene la cantidad porcentual de adición del biosólido. Desde el punto de vista ambiental se evaluó la inocuidad de los metales presentes en el biosólido, desarrollando la prueba Toxicity Characteristic Leaching Procedure (TCLP) en ladrillos fabricados con adición del residuo.
\end{abstract}

Palabras clave: propiedades mecánicas, biosólido, materiales de construcción, resistencia a la compresión, arcilla, mezclas biosólido-suelo.

* $\quad$ M. Sc. Ing. Ambiental. Ing. civil. Docente tiempo Completo Universidad Santo Tomás, investigador del grupo ACBI “Ambiental, Civil y Básicas en investigación” de la Universidad Santo Tomas - Tunja. Boyacá-Colombia. Correo: william.mozo@usantoto.edu.co

** M. Sc. Ing. Materiales y Procesos. Técnico operativo. Laboratorio de Caracterización de Materiales. Departamento de Materiales y Minerales. Facultad de Minas. Universidad Nacional de Colombia. Sede Medellín, Correo: aagomezz@unal.edu.co

*** Postulante a doctora en Desarrollo Sostenible. M. Sc. Ing. civil, Área Ambiental. Ing. química. Profesora asistente, Escuela de Ingeniería Ambiental, Facultad de Ingeniería, líder Grupo Geomática y Ambiente - GIGA, Universidad Pedagógica y Tecnológica de Colombia, Tunja, Boyacá Colombia, correo: gloria.camargo@uptc. edu.co 


\section{Effect of adding biosolids (dry) to a ceramic paste on mechanical strength of bricks}

\section{Abstract}

Sewage sludge was added as part of ceramic slurry for making bricks. Raw materials (clay and sludge) were characterized in terms of chemical composition, mineralogical phases, thermal behavior and physical characteristics. Test tiles with up to $15 \%$ inclusion of sludge in the dry state of standard dimensions according Norma Técnica Colombiana (NTC) 296 and calcined at temperatures of $950^{\circ} \mathrm{C}, 1000{ }^{\circ} \mathrm{C}$ and $1050{ }^{\circ} \mathrm{C}$. In accord of the NTC 4017, compression resistance test, were performed in order to evaluate the effect on this property has the percentage amount of addition of the sludge. From environmental point of view, the safety of metals in sludge was evaluated by developing the Toxicity Characteristic Leaching Procedure (TCLP) test bricks made with the addition of the residue.

Keywords: mechanical properties, sewage, sludge, construction materials, biosolid soil mix. 


\section{INTRODUCCIÓN}

La disposición del biosólido en rellenos sanitarios es una de las formas más comunes para deshacerse de este material. Esta práctica puede generar lixiviados lo que podría causar problemas de eutroficación y contaminación por nitratos en los mantos acuíferos [1], contaminación de las aguas superficiales por escorrentía, contaminación de los suelos por un elevado contenido de metales pesados, presencia de olores desagradables y problemas de salud pública [2].

Uno de los objetivos planteados en la reunión de los países miembro de las Naciones Unidas en el año 2000 [3] fue: "Garantizar el sustento del medio ambiente", y en consecuencia, el presente estudio tiene como fin contribuir al cumplimiento de dicho compromiso, evaluando la posibilidad de incorporar biosólido proveniente de una planta de tratamiento de aguas residuales como materia prima en la pasta cerámica tradicional para la fabricación de ladrillos.

Lin y Weng [4] estudiaron la fabricación de ladrillos usando como materias primas arcilla y cenizas de lodo de agua residual. Demostraron que la incorporación de la ceniza en la mezcla disminuye el índice de plasticidad y la contracción por secado de los elementos moldeados. Las cenizas fueron usadas como sustituto parcial de la arcilla, y los porcentajes usados son de hasta un $50 \%$ de sustitución.

Weng y Lin [5] incorporaron lodos secos de una planta de tratamiento de aguas residuales. En la investigación determinaron que la resistencia a la compresión disminuye en la medida en que el porcentaje de incorporación de lodo, aumenta.

Ismail [6] evaluó el efecto de residuos orgánicos como aserrín, tabaco y pasto sobre las propiedades de ladrillos cocidos y sin cocer. Concluyó que el hecho de incluir estos residuos orgánicos dentro de la pasta cerámica tiene consecuencias como la necesidad de una mayor cantidad de agua para el proceso de extrusión y un incremento (proporcional al porcentaje de residuo) de la contracción de secado y total.

Factores como la cantidad de residuo, el aumento en número de las plantas de tratamiento de aguas residuales (PTAR) [7] y una menor cantidad de lugares de disposición normal, temporal o de emergencia, motivaron el desarrollo de la investigación que dio origen a este artículo y la búsqueda de nuevas alternativas de aprovechamiento.

\section{MATERIALES Y MÉTODOS}

Las materias primas utilizadas fueron arcilla y biosólido. La arcilla proviene de una empresa ladrillera de Boyacá en Colombia; el biosólido corresponde a un subproducto 
proveniente de la PTAR El Salitre (Bogotá, D. C.). Los afluentes en la PTAR son de origen doméstico principalmente. Actualmente la PTAR entrega $4000 \mathrm{l} / \mathrm{s}$ de agua tratada al río Bogotá [8], y como producto de esta actividad se tiene un promedio de producción de 4500 toneladas de biosólido al mes [9].

\subsection{Caracterización de materias primas}

La composición química de las materias primas fue obtenida mediante la técnica de espectroscopia de energía dispersiva (EDS por sus siglas en inglés) por medio de una sonda marca OXFORD acoplada a un microscopio electrónico de barrido (SEM por sus siglas en inglés) marca JEOL referencia 5910 JSM. La composición mineralógica se detectó a través de la técnica de difracción de rayos X (DRX) en un equipo difractómetro de Rayos X marca PANalytical, referencia X'Pert PRO MPD, utilizando una radiación de cobre (longitud de onda 1,5406 Å) proveniente de la polarización de una fuente cerámica de este material con un voltaje de $45 \mathrm{kV}$ y una corriente de $40 \mathrm{~mA}$. Se hizo un barrido en el eje 2 theta entre $5^{\circ}$ y $70^{\circ}$ con un paso de $0,013^{\circ}$ y un tiempo por paso de $59 \mathrm{~s}$. El comportamiento térmico de los materiales se determinó con base en la técnica de análisis térmico simultáneo (STA por sus siglas en inglés) en un equipo marca Netzsch referencia STA 409 CD, sometiendo los materiales a un programa controlado de temperatura y tiempo (temperatura ambiente hasta $1000^{\circ} \mathrm{C}$ a razón de $10^{\circ} \mathrm{C}^{*} \mathrm{~min}^{-1}$ ). La arcilla se caracterizó físicamente determinando la composición granulométrica, la plasticidad, la gravedad específica y la humedad natural, y para las combinaciones de arcilla (Ar) - biosólido (B) propuestas (100\% Ar - 0\% B; $95 \% \mathrm{Ar}-5 \% \mathrm{~B} ; 90 \%$ $\mathrm{Ar}-10 \% \mathrm{~B} ; 85 \% \mathrm{Ar}-15 \% \mathrm{~B}$ ), se determinaron las mismas propiedades que en la arcilla, excepto contenido de materia orgánica y humedad natural.

\subsection{Variables de proceso}

La humedad óptima para la preparación de la pasta cerámica fue establecida en un $19 \%$, producto de los resultados obtenidos en el ensayo de humedad óptima de compactación. Las materias primas $(\mathrm{Ar}-\mathrm{B})$ fueron sometidas a un proceso de molienda $\mathrm{o}$ reducción de tamaño; seleccionando partículas entre $70 \mu \mathrm{m}-1 \mathrm{~mm}$ (material que pasa tamices N. ${ }^{\circ} 18-\mathrm{N} .^{\circ} 200$ ). La dosificación de los materiales en las mezclas se realizó en peso. Los elementos cerámicos fueron conformados con dimensiones: longitud 100 $\mathrm{mm}$, ancho $50 \mathrm{~mm}$, alto $50 \mathrm{~mm}$; según NTC 296 (dimensiones modulares de ladrillos cerámicos). Para el secado, los elementos cerámicos en verde se dejaron a temperatura ambiente, $60^{\circ} \mathrm{C}$ y $105^{\circ} \mathrm{C}$ durante $12 \mathrm{~h}$, con el fin de asegurar un secado adecuado. El proceso de cocción se hizo en un horno mufla de laboratorio, cuya curva de cocción se muestra en la figura 1, compuesta por 3 rampas de temperatura con una tasa de incremento de $5{ }^{\circ} \mathrm{C}^{*} \mathrm{~min}^{-1}$ cada una. 


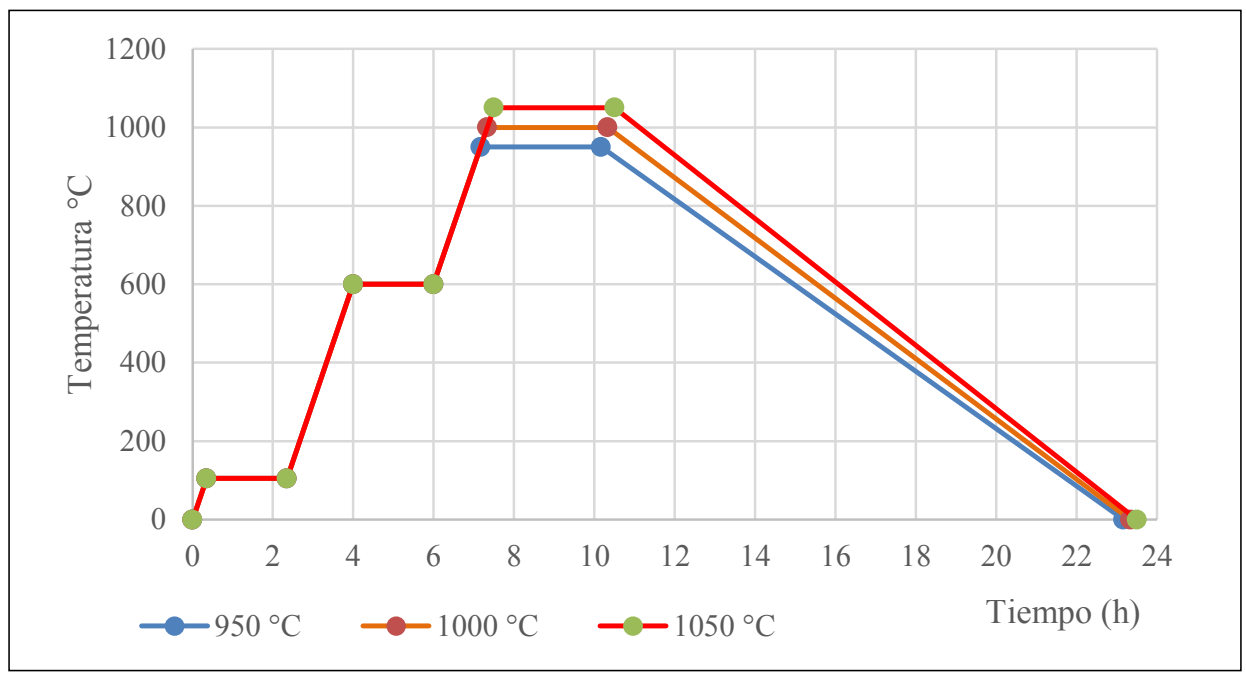

Figura 1. Curvas de cocción

Fuente: elaboración propia

\subsection{Ensayos de propiedades físicas y evaluación ambiental}

A los elementos fabricados se les practicaron ensayos de tasa inicial de absorción de agua, y de absorción de agua y resistencia a la compresión, según la norma NTC 4017 para verificar el cumplimiento de las exigencias en cuanto a utilización como unidades de mampostería de arcilla cocida (NTC 4205). La evaluación ambiental consistió en determinar la movilidad de contaminantes con base en el análisis TCLP siguiendo los lineamientos establecidos por la EPA (Environmental Protection Agency) en su Method 1311, prueba aplicada al elemento fabricado con el mayor porcentaje de inclusión de biosólido y a temperatura de $1050{ }^{\circ} \mathrm{C}$, teniendo en cuenta que para este, se esperaba encontrar la mayor concentración de metales pesados y, por ende, podría generar mayor toxicidad.

\section{RESULTADOS Y DISCUSIÓN}

\subsection{Propiedades físicas}

Los valores de límite líquido, límite plástico e índice de plasticidad (límites de Atterberg) se muestran en la tabla 1. Los resultados demuestran que las mezclas se clasifican como arcillas inorgánicas de media plasticidad y que un incremento en la proporción de biosólido hace que la mezcla tienda a clasificarse como un suelo orgánico de baja plasticidad. El incremento de porcentaje de biosólido se traduce en una dismi- 
nución de plasticidad (índice de plasticidad), comportamiento que permite clasificar al biosólido como un material con propiedades desgrasantes; materiales cuyo fin es disminuir la plasticidad excesiva en las arcillas. [10]

Tabla 1. Caracterización de las mezclas (INV E-125-07)

\begin{tabular}{|l|c|c|c|c|}
\hline \multirow{2}{*}{\multicolumn{1}{|c|}{ Propiedad }} & \multicolumn{4}{|c|}{ Mezclas } \\
\cline { 2 - 5 } & $100 \% \mathrm{Ar} 0 \%$ & $95 \% \mathrm{Ar}$ & $90 \% \mathrm{Ar}$ & $85 \% \mathrm{Ar}$ \\
& $\mathrm{B}$ & $5 \% \mathrm{~B}$ & $10 \% \mathrm{~B}$ & $15 \% \mathrm{~B}$ \\
\hline Límite líquido (\%) & 38,9 & 39,8 & 40,2 & 42,4 \\
\hline Limite plástico (\%) & 18,7 & 20,2 & 23,1 & 25,7 \\
\hline Índice de plasticidad (\%) & 20,2 & 19,6 & 17,1 & 16,6 \\
\hline Gravedad Específica & 2,63 & 2,54 & 2,51 & 2,44 \\
\hline Cont. Mat. Orgánica (\%) & 1,54 & - & - & - \\
\hline Humedad natural (\%) & 17,37 & - & - & - \\
\hline
\end{tabular}

Fuente: elaboración propia

Los datos que se muestran en la tabla 2 corresponden al resultado del ensayo para la determinación de la humedad óptima de compactación. De su análisis es posible concluir que la densidad disminuye con el aumento del porcentaje del biosólido; comportamiento que se atribuye a la menor densidad que tienen las partículas de biosólido en comparación con las de arcilla, lo que se confirma con los datos de gravedad específica mostrados en la tabla 1.

Tabla 2. Contenido óptimo de humedad (INVE - 142-07)

\begin{tabular}{|c|c|c|c|c|}
\hline \multirow{2}{*}{ Propiedad } & \multicolumn{4}{|c|}{ Mezclas } \\
\cline { 2 - 5 } & $100 \% \mathrm{Ar}$ & $95 \% \mathrm{Ar}$ & $90 \% \mathrm{Ar}$ & $85 \% \mathrm{Ar}$ \\
& $0 \% \mathrm{~B}$ & $5 \% \mathrm{~B}$ & $10 \% \mathrm{~B}$ & $15 \% \mathrm{~B}$ \\
\hline Optima de compactación (\%) & 16,5 & 18 & 20 & 21,5 \\
\hline Densidad Seca $(\mathrm{g} / \mathrm{cm} 3)$ & 1,8 & 1,7 & 1,6 & 1,5 \\
\hline
\end{tabular}

Fuente: elaboración propia

El contenido de arenas, limos y arcillas (obtenido del ensayo de granulometría por hidrómetro) para las materias primas arcilla y biosólido se puede ver en la tabla 3. 
Tabla 3. Granulometría (INV E-124-07)

\begin{tabular}{|l|c|c|c|}
\hline \multicolumn{1}{|c|}{ Materia Prima } & \% Arena & \% Limos & \% Arcilla \\
\hline Arcilla & 28 & 32 & 40 \\
\hline Biosólido & 65.06 & 18.94 & 15.99 \\
\hline
\end{tabular}

Fuente: elaboración propia

El material identificado como arcilla (Ar) se clasificó en el triángulo textural de la (USDA - United States Department of Agriculture), y arrojó como resultado un suelo considerado como arcilla: material rico en partículas finas y con un comportamiento plástico al contacto con el agua. Por otra parte, el biosólido por su textura es clasificado como un material franco arenoso [8], lo que significa que el "material contiene mucha arena, pero con suficiente limo y arcilla para aportarle un poco de cohesión" [11], lo que confirma su característica de material desgrasante que disminuye la plasticidad.

\subsection{Composición química}

Los resultados de la composición química elemental (\% en peso) de las materias primas (arcilla, biosólido) se pueden ver en la tabla 4.

Tabla 4. Composición química de las materias primas

\begin{tabular}{|l|c|c|c|c|c|c|c|}
\hline \multirow{2}{*}{ Muestra } & \multicolumn{7}{|c|}{ Elemento } \\
\cline { 2 - 8 } & $\mathrm{O}$ & $\mathrm{Al}$ & $\mathrm{Si}$ & $\mathrm{Fe}$ & $\mathrm{K}$ & $\mathrm{Ca}$ & $\mathrm{C}$ \\
\hline Arcilla & 49,23 & 8,75 & 38,72 & 6,63 & - & - & - \\
\hline Biosólido & 31,40 & 4,35 & 8,65 & 6,33 & 0,92 & 2,81 & 46,02 \\
\hline
\end{tabular}

Fuente: elaboración propia

El elemento que se encuentra en mayor cantidad en la arcilla es el silicio ( $\mathrm{Si}$ ) con un $38,72 \%$, propio de un material arcilloso común para la fabricación de cerámica tradicional; para el biosólido, el elemento que se encuentra en mayor cantidad es el carbono (C) con un 46,02\%, valor atribuible al alto contenido de materia orgánica presente en el mismo.

\subsection{Composición mineralógica}

En las siguientes figuras se observan los difractogramas para las muestras analizadas; en el diagrama en forma de torta, se puede ver la distribución porcentual semi-cuantificada de las fases mineralógicas detectadas en ellas. 


\subsubsection{DRX arcilla}

En la figura 2 se observa que la muestra de arcilla corresponde a un material con alto contenido de cuarzo y de mineral de caolinita, lo que la hace un material arcilloso apto para la fabricación de piezas cerámicas tradicionales y materiales de construcción entre medianas y altas especificaciones (ladrillos, tejas, mampostería, entre otros) [10].

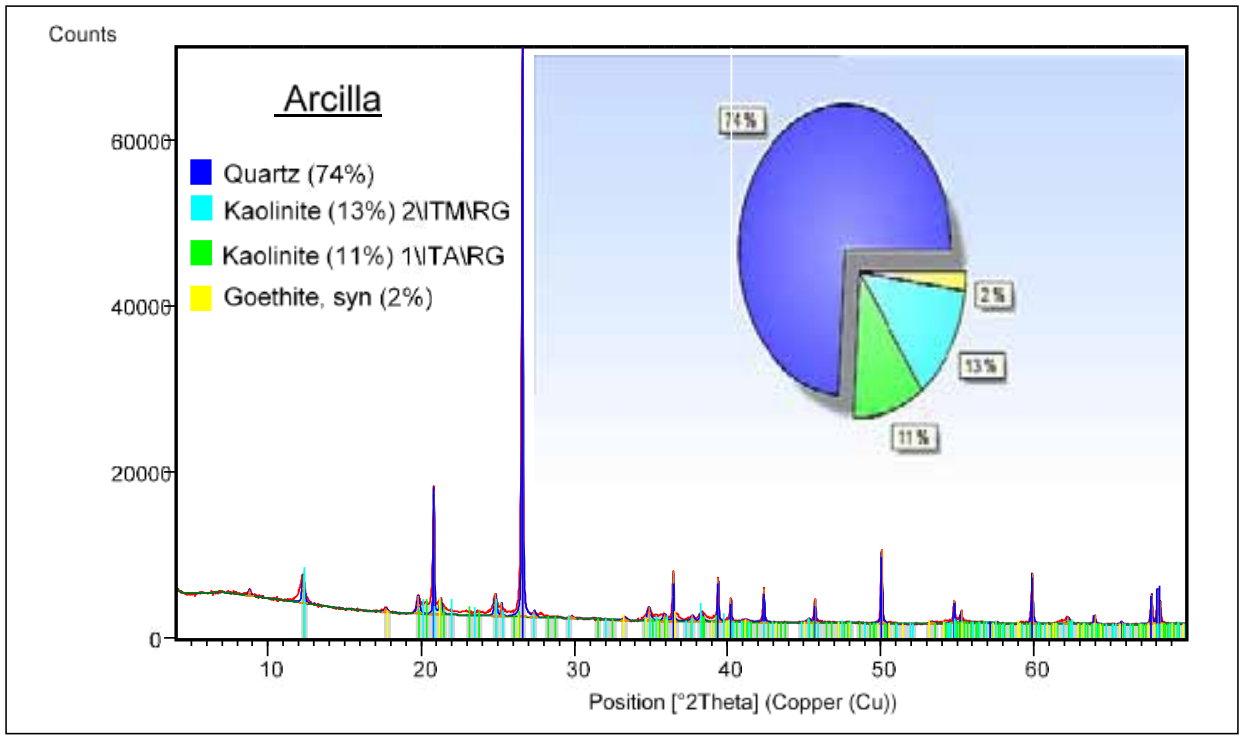

Figura 2. DRX para la arcilla

Fuente: elaboración propia

El contenido de óxidos de hierro (goetita) es bajo y, por ende, la coloración de los elementos fabricados con esta arcilla tiende a tornarse de un color pardo claro, como se evidenció con los ladrillos fabricados para esta investigación.

\subsubsection{DRX biosólido PTAR}

La figura 3 deja ver un contenido $(\approx 20 \%)$ entre las fases mineralógicas gibsita y calcita de la muestra biosólido, que lo hace un material apto para utilizarse como cementante para el sector de la construcción. La presencia de caolinita en un 9,9\%, aunque no sea el mineral más representativo en el biosólido, sugiere posibilidades de aplicación en ladrillos cerámicos, ya que favorece la sinterización al ser activado térmicamente. 


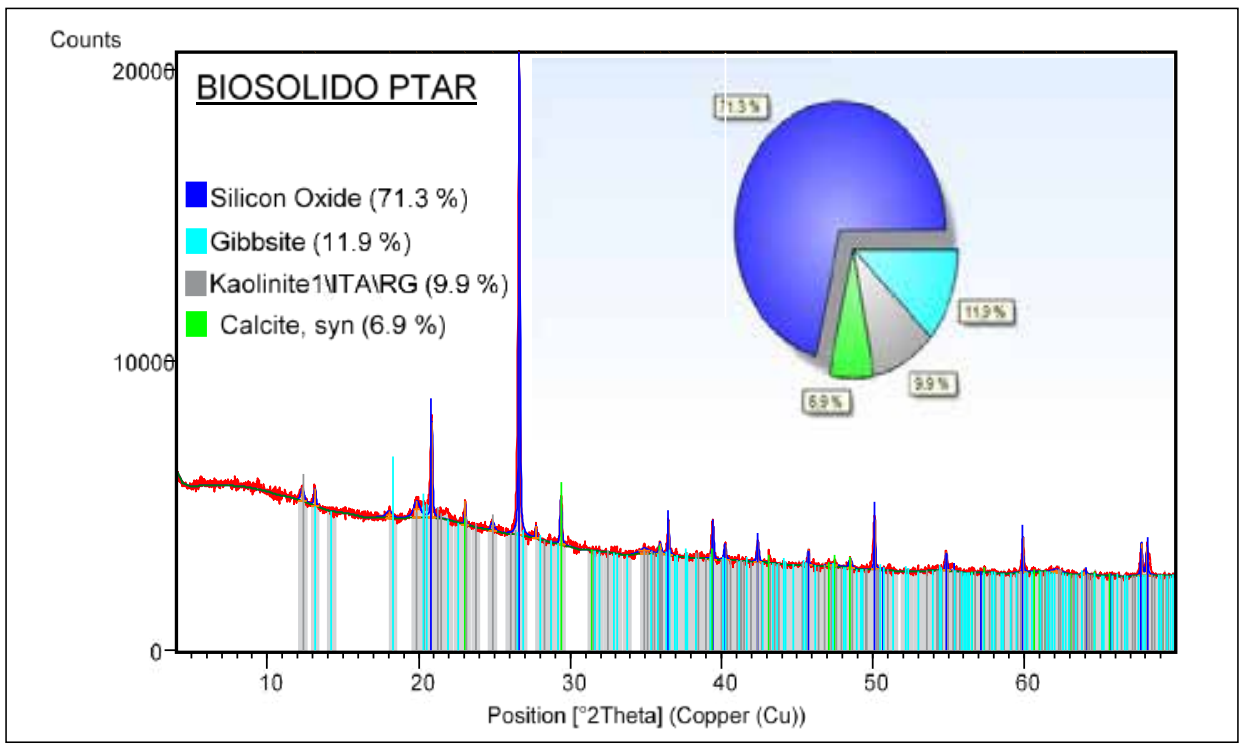

Figura 3. DRX para el biosólido

Fuente: elaboración propia

\subsection{Análisis térmico}

En las figuras 4 y 5 se ven los termogramas para las muestras de las materias primas, donde se observan los picos o cambios térmicos que ocurrieron en su interior. En este análisis térmico se puede ver un primer pico endotérmico a los $58,2^{\circ} \mathrm{C}$, el cual puede estar relacionado con la eliminación del agua libre de la arcilla. El punto de inflexión u onset a $139{ }^{\circ} \mathrm{C}$ puede estar asociado con una pequeña transformación del cuarzo libre presente en la muestra. El pico endotérmico a $293^{\circ} \mathrm{C}$ corresponde a la transformación o transición de la fase goetita a la fase hematita. El pico endotérmico a $565,4^{\circ} \mathrm{C}$ y el exotérmico a $948,3{ }^{\circ} \mathrm{C}$ corresponden a transformaciones de la caolinita, aunque el pico de $565,4^{\circ} \mathrm{C}$ está muy cercano al de la transformación de cuarzo $\alpha$ a cuarzo $\beta$ (573 ${ }^{\circ} \mathrm{C}$ teórico). Estos valores pueden estar ligeramente o por encima o por debajo de los teóricos, debido a que estos pueden estar reportados para la fase mineralógica pura y no para una mezcla de ellas. 


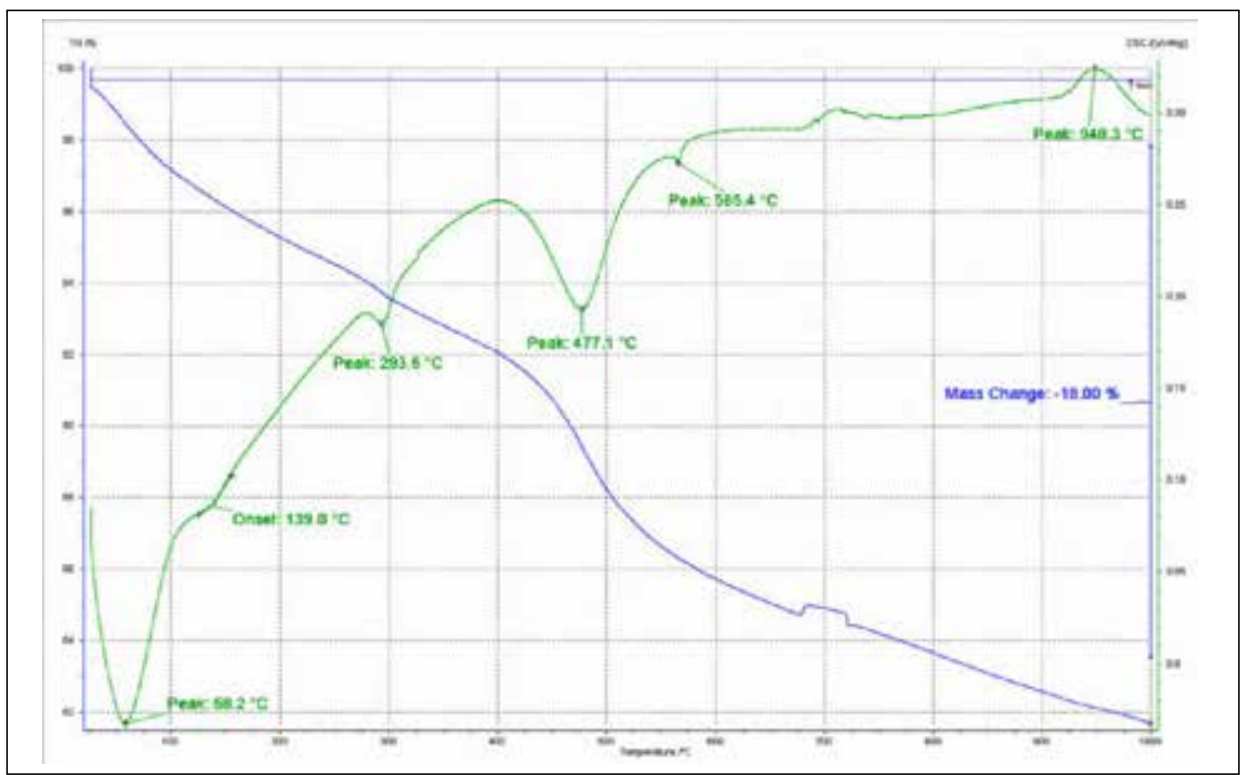

Figura 4. Curvas DSC-TG para la arcilla Fuente: elaboración propia

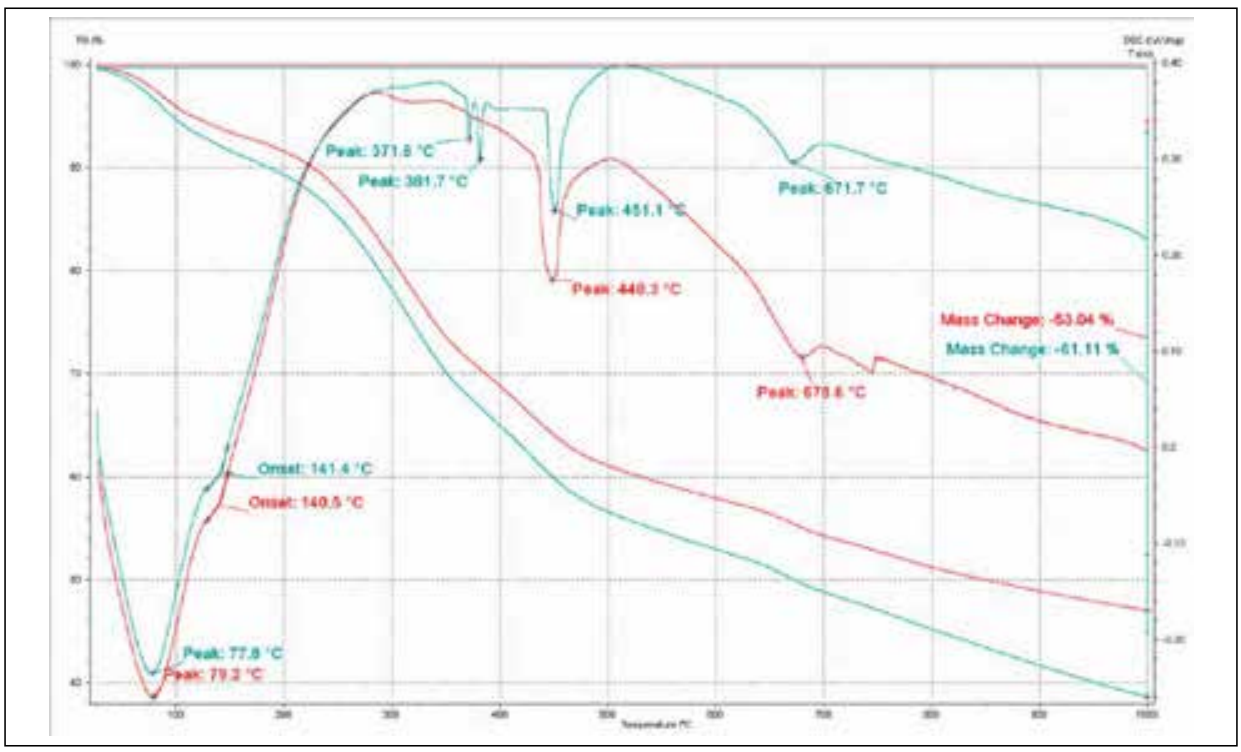

Figura 5. Curvas DSC-TG para el biosólido Fuente: elaboración propia 
Para comprobar la homogeneidad de la muestra de biosólido se realizaron dos ensayos de análisis térmico simultáneo de la misma, en los que se detectaron varios picos. Un pico endotérmico a los $78^{\circ} \mathrm{C}$ está relacionado con la eliminación de agua libre en las muestras. Un punto de inflexión u onset a los $140{ }^{\circ} \mathrm{C}$ puede estar asociado con una pequeña transformación del cuarzo libre presente en las muestras. El doble pico, a $371,6{ }^{\circ} \mathrm{C}$ y $381,7^{\circ} \mathrm{C}$, puede deberse a algún tipo de contaminante de origen polimérico mezclado y no detectado en la muestra. El pico endotérmico alrededor de los $450{ }^{\circ} \mathrm{C}$ podría estar asociado a una transición del mineral de carbonato de calcio de la muestra. Estos valores pueden estar ligeramente o por encima o por debajo de los teóricos, debido a que estos pueden estar reportados para la fase mineralógica pura y no para una mezcla de ellas.

\subsection{Evaluación técnica y ambiental}

\subsubsection{Caracterización biosólido}

El Ministerio de Vivienda, Ciudad y Territorio expidió el Decreto 1287 de 2014, en el cual se establecen criterios (químicos y microbiológicos) para el aprovechamiento de los biosólidos generados en PTAR municipales. En la tabla 5 se presentan dos categorías de clasificación según contenido de metales pesados (Decreto 1287/14) y los valores medios obtenidos entre los años 2005 a 2012 para metales pesados en muestreos realizados periódicamente por la PTAR El Salitre. Los biosólidos de la PTAR El Salitre se clasifican en la categoría B; lo anterior debido al valor encontrado en el cadmio $\left(8,12 \mathrm{~m}^{*} \mathrm{1}^{-1}\right)$, el cual supera los límites establecidos para categorizarlo en A. Para los patógenos no se hizo comparación, debido a que se realizan procesos de digestión y deshidratación al lodo, con el fin de estabilizarlo, adquiriendo características de biosólido. Adicionalmente, para el aprovechamiento del material en la fabricación de ladrillos, se le realiza un proceso de cocción a una temperatura mayor a los $900^{\circ} \mathrm{C}$, lo que garantiza la inactivación de estos microorganismos.

Tabla 5. Criterios químicos-metales decreto 1287 de 2014 - valores medios PTAR EL SALITRE

\begin{tabular}{|c|c|c|c|c|}
\hline \multirow{2}{*}{ Parámetro } & \multirow{2}{*}{ Unidades } & \multicolumn{2}{|c|}{$\begin{array}{c}\text { Decreto 1287/2014 } \\
\text { Criterios biosólidos Ptar }\end{array}$} & $\begin{array}{c}\text { Ptar el salitre Bogotá } \\
\text { Concentración media } \\
\text { años (2005 - 2012) }\end{array}$ \\
\cline { 3 - 4 } & & Categoría A & Categoría B & 14,22 \\
\hline Arsénico & $\mathrm{mg} \mathrm{As}^{*} \mathrm{Kg}^{-1}(\mathrm{BS})$ & 20 & 40 & 8,17 \\
\hline Cadmio & $\mathrm{mg} \mathrm{Cd} * \mathrm{Kg}^{-1}(\mathrm{BS})$ & 8 & 40 & 188,45 \\
\hline Cobre & $\mathrm{mg} \mathrm{Cu}^{*} \mathrm{Kg}^{-1}(\mathrm{BS})$ & 1000 & 1750 & 86,39 \\
\hline Cromo total & $\mathrm{mg} \mathrm{Cr}^{*} \mathrm{Kg}^{-1}(\mathrm{BS})$ & 1000 & 1500 & 4,34 \\
\hline Mercurio & $\mathrm{mg} \mathrm{Hg}^{*} \mathrm{Kg}^{-1}(\mathrm{BS})$ & 10 & 20 & \\
\hline
\end{tabular}




\begin{tabular}{|c|c|c|c|c|}
\hline \multirow{2}{*}{ Parámetro } & \multirow{2}{*}{ Unidades } & \multicolumn{2}{|c|}{$\begin{array}{c}\text { Decreto 1287/2014 } \\
\text { Criterios biosólidos Ptar }\end{array}$} & $\begin{array}{c}\text { Ptar el salitre Bogotá } \\
\text { Concentración media } \\
\text { años (2005 - 2012) }\end{array}$ \\
\cline { 3 - 5 } & & Categoría A & Categoría B & SD \\
\hline Molibdeno & $\mathrm{mg} \mathrm{Mo} * \mathrm{Kg}^{-1}(\mathrm{BS})$ & 18 & 75 & 5,89 \\
\hline Níquel & $\mathrm{mg} \mathrm{Ni} \mathrm{Kg}^{-1}(\mathrm{BS})$ & 80 & 420 & 86,92 \\
\hline Plomo & $\mathrm{mg} \mathrm{Pb} \mathrm{Kg}^{-1}(\mathrm{BS})$ & 300 & 400 & 12,66 \\
\hline Selenio & $\mathrm{mg} \mathrm{Se}^{*} \mathrm{Kg}^{-1}(\mathrm{BS})$ & 36 & 100 & 1078,96 \\
\hline Zinc & $\mathrm{mg} \mathrm{Zn}^{*} \mathrm{Kg}^{-1}(\mathrm{BS})$ & 2000 & 2800 & \\
\hline
\end{tabular}

SD: Sin Dato

BS: Base Seca

\subsubsection{Propiedades técnicas según NTC 4205}

A continuación se presentan los valores promedio de 5 unidades ensayadas bajo los lineamientos de la NTC 4017 [12], para propiedades como tasa inicial de absorción de agua (figura 6), resistencia a la compresión (figura 7) y absorción de agua (figura 8 y 9). Cada propiedad se evalúa con base en las exigencias que se especifican en la NTC 4205 [13]. Para evaluar las anteriores propiedades fue necesario elaborar 120 especímenes.

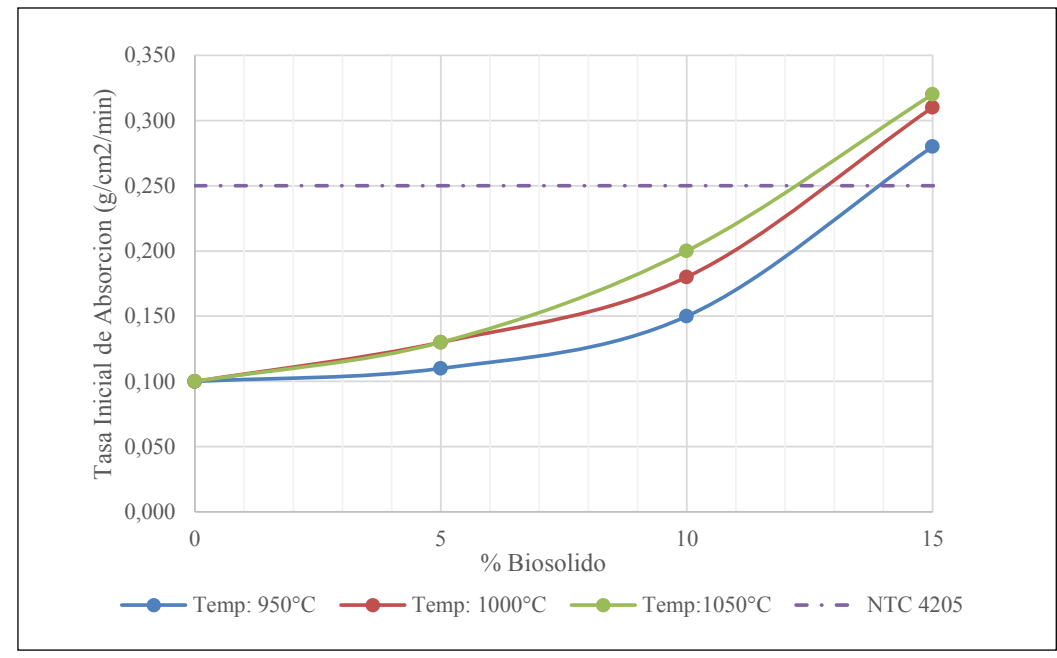

Figura 6. Tasa inicial de absorción Fuente: elaboración propia

Tasa inicial de absorción: se puede evidenciar un incremento en la tasa inicial de absorción con el aumento de biosólido en la mezcla. Lo anterior es un indicio de mayor porosidad, acorde con el espacio que deja la materia orgánica en la matriz cerámica al ser quemada. Como se puede ver en la figura 6 , las unidades de mampostería con- 
formadas ( $85 \%$ Ar-15\% B) no cumplen con los requerimientos mínimos establecidos por la norma, ya que presentan valores que se encuentran entre $0.28 \mathrm{~g} / \mathrm{cm} 2 / \mathrm{min}$ y 0.32 $\mathrm{g} / \mathrm{cm} 2 / \mathrm{min}$, superiores a $0.25 \mathrm{~g} / \mathrm{cm} 2 / \mathrm{min}$, valor máximo permitido por la norma. En la norma técnica NTC 4205 se recomienda que para la utilización de estas unidades "se debe vigilar el cumplimiento de los requisitos de absorción y resistencia, y diseñar los morteros de pega y tiempos de pre-humedecimiento apropiados". Esto debido a que la cantidad de agua que la unidad de mampostería absorbe puede afectar el fraguado normal del mortero, generando consecuencias como su pérdida de resistencia. Vale la pena aclarar que el hecho de que no cumpla con los requisitos de tasa inicial de absorción de agua no demerita la calidad del elemento cerámico obtenido.

Resistencia a la compresión: las dos variables manipuladas en la investigación tienen influencia directa en la resistencia a la compresión. Con la temperatura existe una relación directamente proporcional y con \% de biosólido inversamente proporcional. Según la NTC 4205, unidades de mampostería no estructural deben cumplir con resistencias mínimas a la compresión de 14 MPa y unidades de mampostería estructural de $20 \mathrm{MPa}$. Todos los elementos cerámicos elaborados con las diferentes combinaciones de temperatura y biosólido cumplen con el requisito mínimo de resistencia a la compresión para ser clasificados como mampostería no estructural. Los elementos cocidos a $1000{ }^{\circ} \mathrm{C}$ y $1050{ }^{\circ} \mathrm{C}$ con los diferentes porcentajes de biosólido $(0 \%-5 \%$ $10 \%-15 \%$ ) cumplen con los requerimientos de la norma para ser clasificados como mampostería estructural.

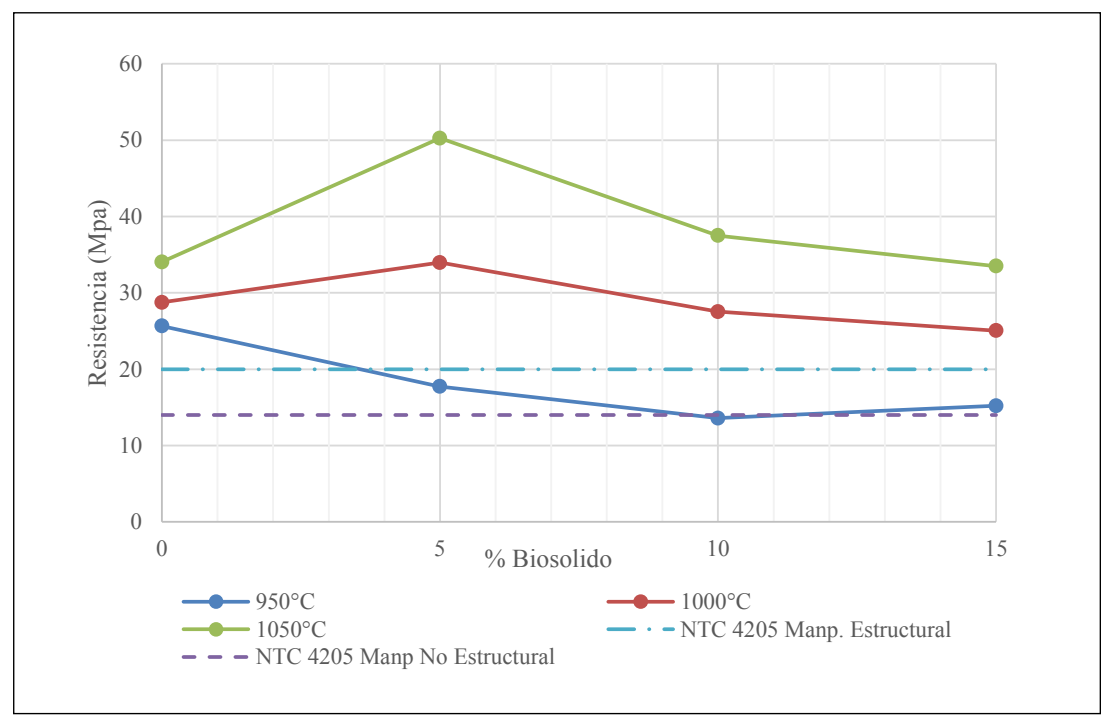

Figura 7. Resistencia a la compresión

Fuente: elaboración propia 
Absorción de agua: los resultados muestran que la absorción de agua aumenta con el incremento en la adición del biosólido a la pasta cerámica, y disminuye con el aumento en la temperatura, comportamiento directamente ligado a la finalización del proceso de vitrificación y el sellado de los poros abiertos debido a la cocción.

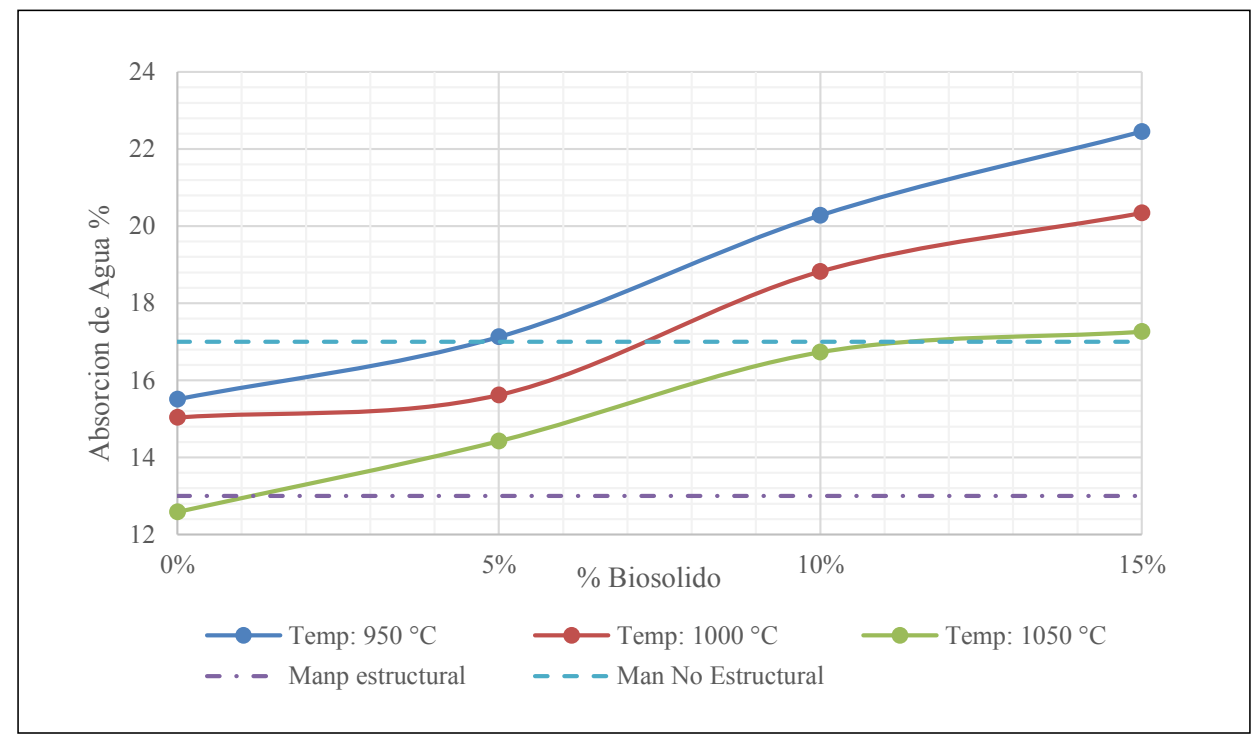

Figura 8. Absorción de agua-uso interior Fuente: elaboración propia

La NTC 4205 establece los requisitos que deben cumplir las unidades de mampostería para uso interior y exterior así: mampostería estructural (13\%) y mampostería no estructural (17\%). Con base en los resultados obtenidos pueden ser usados como mampostería no estructural en interiores los elementos cerámicos que fueron elaborados con un $5 \%$ de adición de biosólido en cualquiera de las tres temperaturas de cocción, así como elementos cocidos a $1050{ }^{\circ} \mathrm{C}$ con hasta el $15 \%$ de biosólido. Respecto al uso en exteriores, en la figura 9 se puede ver que ningún elemento de los elaborados cumple con los valores exigidos en la norma. 


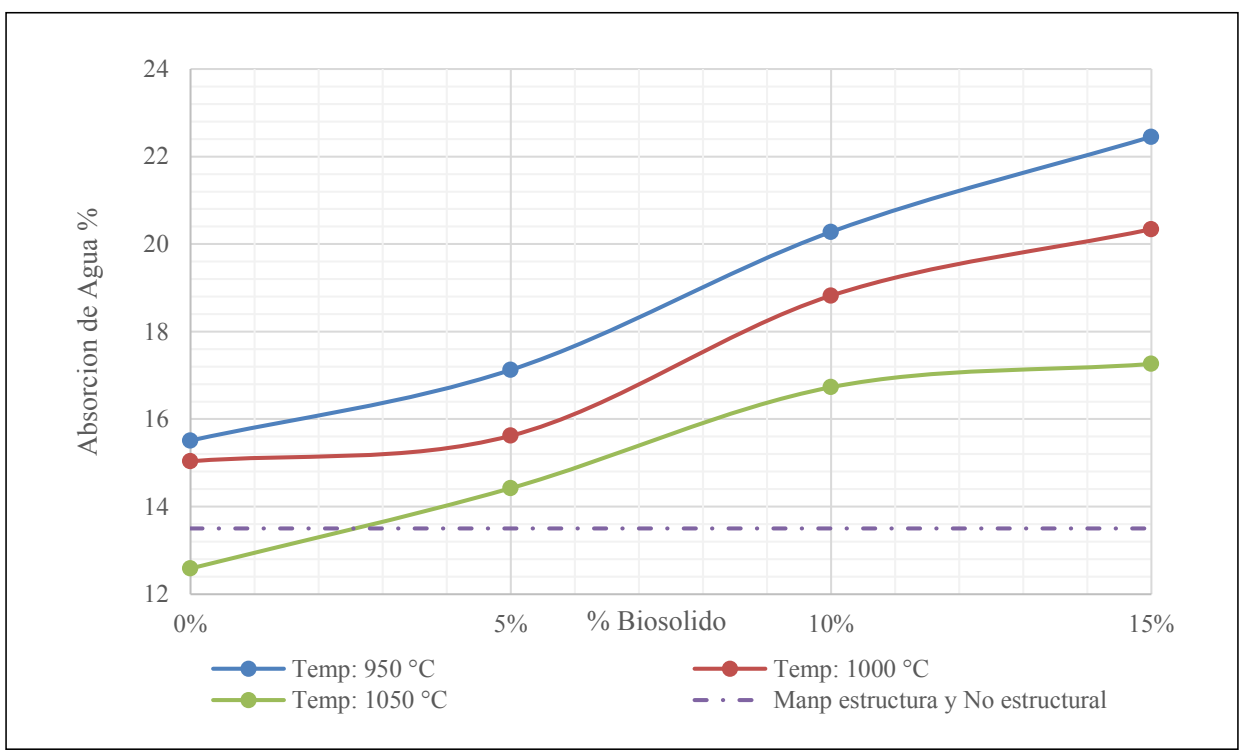

Figura 9. Absorción de Agua - uso exterior Fuente: elaboración propia

\subsection{3 (TCLP) Biosólido}

Para estimar la inocuidad de los metales pesados se hizo el ensayo de prueba de lixiviación en el Laboratorio Integrado de Ingeniería Civil y Ambiental de la Universidad de los Andes, para determinar la característica de toxicidad (TCLP) a un elemento elaborado con $15 \%$ de biosólido a una temperatura de $1050{ }^{\circ} \mathrm{C}$. Como se puede ver en la tabla 6 , las concentraciones de metales lixiviados son mucho menores que los límites establecidos en el Decreto 4741 de 2005 [14], en el cual se establece como residuo peligroso o desecho tóxico aquel que: "al realizársele una prueba de lixiviación (TCLP), contiene una concentración superior a los niveles máximos permisibles en el lixiviado". Lo anterior implica que el ladrillo con $15 \%$ de biosólido no se considera un material tóxico, y puede ser empleado como material para mampostería estructural y no estructural, de acuerdo con la norma NTC 4205.

Tabla 6. Análisis TCLP

\begin{tabular}{|l|c|c|c|}
\hline \multicolumn{1}{|c|}{ Metales } & Decreto 4741 de 2005 & $\begin{array}{c}\text { Ladrillo } 15 \% \text { biosólido } \\
1050{ }^{\circ} \mathrm{C}(\mathrm{mg} / \mathrm{l})\end{array}$ & $\begin{array}{c}\text { Taiwan }- \text { EPA } \\
\text { Regulated TCLP limit } \\
(\mathrm{mg} / \mathrm{l})\end{array}$ \\
\hline Arsénico (As) & 5 & $<0,012$ & 5 \\
\hline Bario $(\mathrm{Ba})$ & 100 & 0,056 & 100 \\
\hline
\end{tabular}




\begin{tabular}{|l|c|c|c|}
\hline \multicolumn{1}{|c|}{ Metales } & Decreto 4741 de 2005 & $\begin{array}{c}\text { Ladrillo } 15 \% \text { biosólido } \\
1050^{\circ} \mathrm{C}(\mathrm{mg} / \mathrm{l})\end{array}$ & $\begin{array}{c}\text { Taiwan }- \text { EPA } \\
\text { Regulated TCLP limit } \\
(\mathrm{mg} / \mathrm{l})\end{array}$ \\
\hline Cadmio (cd) & 1 & 0,034 & 1 \\
\hline Cromo Total (Cr) & 5 & $<0,012$ & 5 \\
\hline Mercurio (Hg) & 0,2 & $<0,083$ & 0,2 \\
\hline Plata(Au) & 5 & 0,144 & 5 \\
\hline Plomo $(\mathrm{Pb})$ & 5 & $<0,015$ & 5 \\
\hline Selenio $(\mathrm{Se})$ & 25 & $<0,036$ & 25 \\
\hline
\end{tabular}

Fuente: elaboración propia

\section{CONCLUSIONES}

La incorporación de biosólido en la pasta cerámica tiene efectos como aumento de la resistencia a la compresión, disminución de la plasticidad, reducción de la contracción en el secado y aumento de la porosidad de los elementos cerámicos. El contenido de gibsita y calcita encontrado en el biosólido hace, de este residuo, un material cementante apto para una posible incorporación como materia prima en la obtención de materiales del sector de la construcción.

La propiedad de absorción de agua medida en los elementos obtenidos limita su uso como unidades de mampostería estructural. No obstante, no descalifica el ladrillo en su totalidad, pues es la resistencia a la compresión la propiedad determinante para rechazar un ladrillo.

Elementos cerámicos elaborados con incorporación de biosólido hasta un $10 \%$, pueden ser utilizados según la norma NTC 4205, como mampostería no estructural de uso interior para muros de cierre o divisorios que únicamente atiendan a la carga de su propio peso.

Al aplicar la prueba TCLP, se encontró que las concentraciones para los metales pesados estudiados, están por debajo de los límites máximos establecidos en la normativa de Gestión de Residuos Peligrosos Colombiana (Decreto 4741 de 2005).

Adicionalmente, al realizar el proceso de cocción de ladrillos (vitrificación) se garantiza la inactivación de cualquier microorganismo patógeno que pudiese estar presente en el biosólido, de tal forma que los elementos de mampostería elaborados bajo las condiciones óptimas de este estudio podrán utilizarse de manera segura y no podrán ser considerados como materiales tóxicos. 
Finalmente, con los resultados de esta investigación se ha demostrado que es posible obtener ladrillos que cumplan con estándares de calidad controlando el porcentaje de adición de biosólido y la temperatura de cocción.

\section{AGRADECIMIENTOS}

La presente investigación ha sido posible gracias al apoyo de instituciones de Educación Superior y entidades del sector privado como Universidad Santo Tomas Tunja, Universidad Pedagógica y Tecnológica de Colombia, Universidad Nacional de Colombia - Sede Medellín. Empresa de Acueducto y Alcantarillado de Bogotá y Planta de Tratamiento de Aguas Residuales El Salitre. Los autores presentan sus más sinceros agradecimientos.

\section{REFERENCIAS}

[1] Facultad de ciencias químicas. Universidad Autónoma de Chihuahua. Manejo de sólidos residuales o biosólidos. [Online]. [Citado 15 enero 2012]. En Internet: < http://www.oocities. org/edrochac/residuales/biosolidos9.pdf>

[2] Diputación de Zamora medio ambiente. (2007). Abónate al lodo. Gestión de lodos de depuradoras y fosas sépticas. Zamora, España.

[3] Organización de las Naciones Unidas. Obtenido de Organización de las Naciones Unidas: http://www.un.org/es/millenniumgoals/ (21 de mayo de 2014).

[4] LIN, Deng-Fong y WENG, Chih-Huang. Use of sewage ash as brick material. En Journal of Environmental Engineering. Octubre, 2001, Vol. 127, n. 10.

[5] WENG, Chih-Huang; LIN, Deng-Fong y CHIANG, Pen-Chi. Utilization of sludge as brick materials. En Advances in EnvironmentalResearch. 2003. pp. 679-685.

[6] DEMIR, Ismail. Effect of organic residues addition on the technological properties of clays bricks. En Waste Management. Mayo, 2007, n. ${ }^{\circ} 28$, pp. 622-627.

[7] PTAR SALITRE FASE I. Planta de tratamiento de aguas residuales [online]. [Citado 20 mayo2011]. Disponible en $<$ http://www.acueducto.com.co/wpsv61/wps/html/resources/ PTAR/plegable_general.pdf $>$

[8] Acueducto, Agua y Alcantarillado de Bogotá. 'Planta de Tratamiento de Aguas Residuales El Salitre-Fase I. Informe de actividades diciembre 2011-enero 2012. Bogotá, 2011.

[9] Gestión de subproductos [online]. Bogotá Colombia. PTAR El Salitre [citado 20 mayo 2011]. Disponible en $<$ http://www.acueducto.com.co $>$

[10] MORALES GÜETO, Juan. Tecnología de los materiales cerámicos. Consejería de educación. Comunidad de Madrid. Díaz de Santos, 2005.

[11] CASTRO FRANCO, Hugo E. Fundamentos para el conocimiento manejos de suelos agrícolas. Instituto Universitario Juan de Castellanos, Tunja, 1998. 
[12] INSTITUTO COLOMBIANO DE NORMAS TÉCNICAS Y CERTIFICACIÓN. Ingeniería civil y arquitectura. Métodos de muestreo y ensayos de unidades de mampostería de arcilla. NTC 4017. Bogotá, D. C.: ICONTEC, 2000.

[13] INSTITUTO COLOMBIANO DE NORMAS TÉCNICAS Y CERTIFICACIÓN. Ingeniería civil y arquitectura. Unidades de mampostería de arcilla cocida. Ladrillos y bloques cerámicos. NTC 4205. Bogotá, D. C.: ICONTEC, 2000.

[14] MINISTERIO DE AMBIENTE, VIVIENDA Y DESARROLLO TERRITORIAL. Decreto 4741 de 2005. "Por el cual se reglamenta parcialmente la prevención y manejo de los residuos o desechos peligrosos generados en el marco de la gestión integral”. 30 Dic 2005.

[15] Decreto 1287 del 10 de julio de 2014, por el cual se establecen criterios para el uso de los biosólidos generados en plantas de tratamiento de aguas residuales municipales. Ministerio de Vivienda, Ciudad y Territorio.

[16] MOZO M., William R. Valorización de biosólido de PTAR en la fabricación de ladrillos. Libro proyecto de grado maestría. Tunja. 2014. 